\title{
LOCAL FIBRE ORIENTATION AND FIBRE VOLUME FRACTION MAPPED NUMERICAL MODELS BASED ON X-RAY COMPUTER TOMOGRAPHY SCANS
}

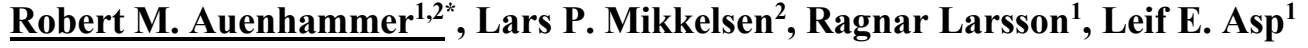 \\ ${ }^{1}$ Industrial and Materials Science, Chalmers University of Technology, SE-41296, Göteborg, Sweden \\ ${ }^{2}$ DTU Wind Energy, Technical University of Denmark, DK-4000 Roskilde, Denmark \\ *robaue@chalmers.se
}

Fibre reinforced composites are inhomogeneous in terms of local polymer/fibre variation. The degree of inhomogeneity is set by the volume fraction, which has a significant influence on the macroscopic material behaviour. Modelling the local material variations is normally challenging due to limited data about the morphology. The standard approach is to upscale the local material response to the macrolevel based on homogenisation. However, the advance in X-ray computer tomography allows for Direct Numerical Simulation of the fully resolved model. In [1] a full process how to create finite element models based on X-ray computer tomography data for non-crimp reinforced polymers was described. The core of this X-ray computer tomography aided process relies on the boundary identification between free polymer resin and fibre bundles as well as a local material orientation. This allows for the investigation of local stress concentrations [2]. As an extension to this, models with mapped local fibre volume fraction are presented. Consequently, an even more accurate local stress distribution can be reflected.

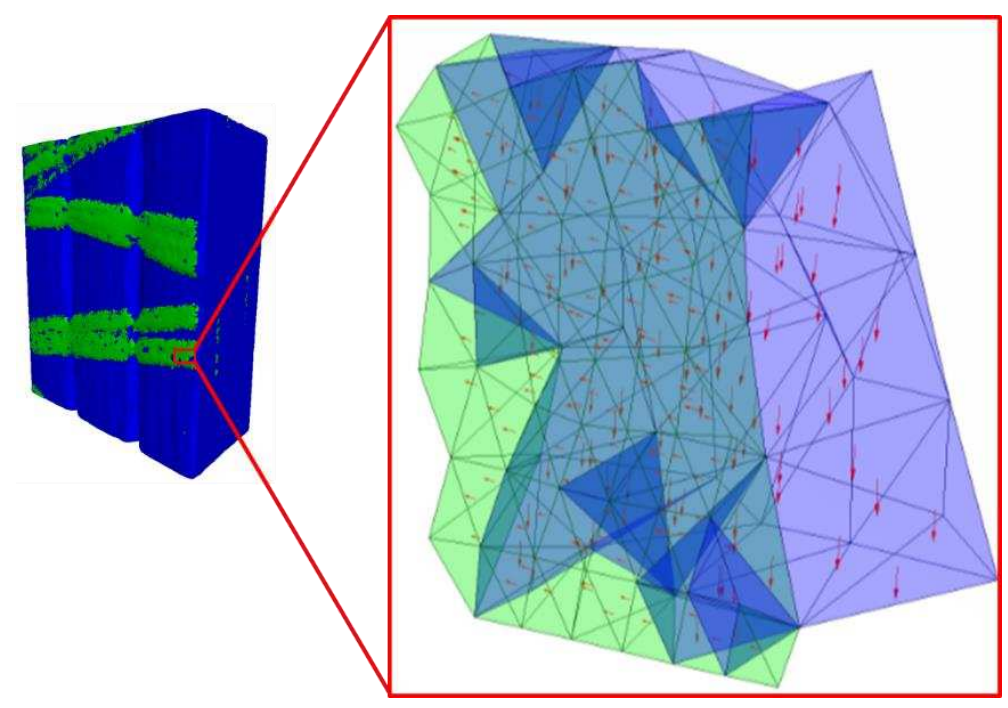

Figure 1. Zoom in to visualise the elementwise material orientation modelling of X-ray computer tomography aided engineering (XAE)

\section{References}

[1] R. M. Auenhammer, L. P. Mikkelsen, L. E. Asp and B. J. Blinzler (2021) Automated x-ray computer tomography segmentation method for finite element analysis of non-crimp fabric reinforced composites, Composite Structures, 256, 113136.

[2] R. M. Auenhammer, L. P. Mikkelsen, L. E. Asp, B. J. Blinzler (2020) X-ray tomography based numerical analysis of stress concentrations in non-crimp fabric reinforced composites - assessment of segmentation methods, IOP Conference Series: Materials Science and Engineering, 942, 012038. 